behind its insertion, so as to weaken its action; he then performs a modified Critchett's three stitch advancement on the opponent, and fixes the ends of the suture to the temple with strapping. The aftertreatment consists of atropin to the eyes and an occlusion bandage for a week, at the end of which time the refraction is estimated and glasses are ordered if necessary.

R. R. JAMES.

\title{
CORRESPONDENCE
}

\section{THE NOTATION OF AXES OF CYLINDERS}

To the Editor of the BRITISH JOURNAL OF OPHTHALMOLOGY. .

SIR,-With reference to Mr. Gray Clegg's interesting article in . the October issue of the Journal, upon the above subject, I may point out that in February, 1910, in a short note to the Lancet, I submitted that the difficulty in regard to the question of notation arose from the fact that there was no standard method. "Every method in vogue is a workable method, but there is no uniformity, because there is no common standard, and because these methods lack the basis of a standard which is recognized by the world, about which no confusion can arise, it is impossible to expect any universal agreement." Such a standard, nevertheless, is available, and that is the mariner's compass. In this compass the poles are marked zero, and the equator, or horizontal line, $90^{\circ}$. With this compass as the standard, the axes of the cylinders are simply required to be indicated as so many degrees from zero in approaching the equator, north-east, or north-west, according as the axis is downwards and outwards, or downwards and inwards. Thus by this means it is possible to avoid the confusion which frequently arises by dealing with degrees higher than 90 . During the past nine years I have found this method very simple, workable, and convenient.

$$
\text { I am, Sir, }
$$

Yours truly,

LONDON, W.

Percy Dunn. 
To the Editor of the British JOURNAL OF OPHTHALMOLOGY.

SIR,-When prescribing spectacles for a patient, the primary object of employing any standard is to enable the patient to get the correct spectacles from an optician. "Paris inches" are no longer employed and in all countries the dioptre is recognized.

When defining the angle at which the cylinder is to be placed, no such uniformity exists. This is due, at least in part, to ophthalmic surgeons having divers views as to what is most important. In a recent article, "The Notation of Cylinder Axes," by J. Gray Clegg (B.J.O., October, 1919) these views and their divergencies are carefully exposed. These can be divided into two classes, those in which the notation is symmetrical about the nose, and those in' which it is similar.

It appears to me that in deciding what method should be adopted, the point most worthy of consideration is what method will enable the optician most easily and accurately to supply the patient with the spectacles the surgeon desires.

From among the many methods employed, the opticians have had - to choose that one which most completely fulfils their requirements. Had they chosen any of the methods relying on symmetrical notation about the mid-line they would have required two machines for marking off the lenses, one for the right lens and one for the left, or alternatively two sets of graduations on the one machine-in either case a very fruitful source of error. The result is that they have decided that they will use a notation that is similar in each eye and in which the zero is horizontally on the left hand side as one faces the patient, and the numbers are marked, increasing from left to right, on the lower part of the frame. This they call the "Standard" notation.

My enquiries lead me to believe that every optician in London, with one exception, employs this method and that when a patient takes a prescription to an optician, the axis is invariably transcribed into this form before it is sent into the workshops to be made up.

In view of the fact that error is less likely to arise if the optician has merely to transcribe the axis rather that translate it, it would appear that it is to the patient's advantage that the surgeon should employ the "Standard" notation.

$$
\text { Yours, etc., }
$$

LONDON, W.

B. T. LANG. 